\title{
A User Experience and eHealth Literacy Inspection of a Lab Test Interpretation Mobile App for Citizens
}

\author{
Helen MONKMAN ${ }^{\mathrm{a}, 1}$, Leah MACDONALD ${ }^{\mathrm{a}}$, Janessa GRIFFITH ${ }^{\mathrm{b}, \mathrm{c}}$, and Blake \\ LESSELROTH $^{\mathrm{d}}$ \\ ${ }^{a}$ School of Health Information Science, University of Victoria, Canada \\ ${ }^{\mathrm{b}}$ Institute for Health System Solutions and Virtual Care, \\ Women's College Hospital, Toronto, Canada \\ ${ }^{c}$ Faculty of Health Sciences, Douglas College, Coquitlam, Canada \\ ${ }^{\mathrm{d}}$ University of Oklahoma-Tulsa, School of Community Medicine, USA
}

\begin{abstract}
People are increasingly able to access their laboratory (lab) results using patient-facing portals. However, lab reports for citizens are often identical to those for clinicians; without specialized training they can be near impossible to interpret. In this study, we inspected a mobile health application (app) that converts traditional lab results into a citizen-centred format. We used the Health Literacy Online (HLO) checklist to inspect the app. Our inspection revealed that most of the app's strengths were related to its Organization of Content and Simple Navigation and most of its weaknesses were related to Engage Users. We also identified several usability and user experience (UX) issues that were beyond the purview of the HLO checklist. Although this app represents an important step towards making lab results universally accessible, we identified several opportunities for improvements that could increase its value to citizens.
\end{abstract}

Keywords. User experience, usability, eHealth literacy, health literacy, laboratory results, blood test results, consumer health informatics, mHealth

\section{Introduction}

Health care professionals use laboratory (lab) tests for screening and diagnosis of health conditions as well as monitoring of therapies and outcomes. Test results are often available to citizens (i.e., health consumers, laypeople, and patients) for review using online portals. However, these digital platforms typically report results in the same format used by health professionals. For example, some have criticized blood test reports for lacking emphasis, clarity, simplicity, relevancy, and ease of use [1]. Moreover, reference ranges often vary between labs, potentially creating confusion [2]. These flaws undoubtedly limit the extent that citizens can understand, contextualize, and use their results. Health-related social needs including social environment, income, and education and literacy invariably potentiate these challenges. As a result, those with limited

\footnotetext{
${ }^{1}$ Helen Monkman, Health Information Science, University of Victoria, PO Box 1700 STN CSC, Victoria BC,V8W 2Y2, Canada, E-mail: monkman@uvic.ca
} 
functional health literacy or numeracy may be unable or unwilling to use these newer consumer-centered technologies [2-4].

Using evidence-based design and user-experience (UX) research methods is important when attempting to improve the usability and usefulness of consumer-facing medical reports. For example, research indicates citizens prefer lab results to include an explanation of the context, a straightforward interpretation of findings, and recommendations to improve their clinical values [5,6]. Simple design modifications, data visualization techniques, and visual metaphors can foster understanding, lead to new insights, and improve situational awareness (e.g., recognizing an urgent issue or general trend [7]). Equipping people with better information can empower them to engage in more effective and person-centered conversations about their health (e.g.,(8-10)]).

One approach to transform lab results into more useful information for citizens is to translate existing lab reports into citizen-centred formats. We identified a mobile application (app) that translates typical clinical reports into more accessible informational artifacts for citizens. In this study, we evaluated this app through the dual lenses of eHealth literacy and usability. The purpose of the research is to (1) examine how well the app met its stated aims; (2) identify opportunities for improvement; and (3) determine whether this app could help educate and empower citizens.

\section{Methods}

The app under inspection seeks to translate traditional lab results into a format that citizens can understand and use by incorporating techniques such as using visuals to depict values, providing context about the test, as well personalized insights (e.g., "your eGFR value may suggest a moderate renal insufficiency"; "it is highly recommended that you inform your doctor about your potassium [K] results") based on the result values. Users input their lab results by scanning them using their smartphone camera or manually enter them. The app integrates several other features and user functions including personal insights based on the user's profile (e.g., recommended schedules for cholesterol and blood pressure tests), vital signs tracking (e.g., weight, heart rate, blood pressure), integration with wearable devices, personalized coaching programs, menstrual cycle tracking, and an active feed of health educational topics. Information is stored locally on the device with the exception that during the translation process it is transmitted to app employees for data extraction verification. Once verified the data is deleted from their system and all features operate offline.

\subsection{Inspection Framework}

We used the US Department of Health and Human Services Office of Disease Prevention and Health Promotion's Health Literacy Online (HLO) Checklist for our inspection protocol and as an analytical framework [11]. Other investigations have also used the HLO Checklist for inspections of eHealth literacy issues (e.g., [12,13]). The HLO Checklist [11] is comprised of 43 items organized into five categories: (1) Write Actionable Content; (2) Display Content Clearly on the Page; (3) Organize Content and Simplify Navigation; (4) Engage Users; and (5) Test your Site with Users With Limited Health Literacy. We did not conduct a simulation test with representative users and therefore omitted the last category for this inspection, leaving 35 items in the checklist. 


\subsection{Procedure}

Two user experience and consumer health experts (HM and JG) and a student trainee (LM) installed the app on their own smartphone devices. After launching the app, users were first presented with a description of the features. Then users agreed to the terms of use. First time users set up a profile that guides the eventual translation logic. For consistency, all evaluators used the same profile information (Canadian, male, born September 30, 1957) and sample data. Users were then directed to a landing page with instructions for use. To use the translation feature, users had to input labs by scanning with their smartphone camera or manually entering data using the onscreen keyboard. Each evaluator attempted to upload a lab report for analysis, but scanning feature was inoperative at the time of this inspection. Therefore, we manually entered a standard set of seven lab results, selected to represent different tests and result values within and beyond reference ranges, taken from a fictitious Canadian sample report.

For each checklist item, evaluators recorded on a spreadsheet whether 1) "yes, the mobile app satisfied the criteria"; 2) "no it did not"; or 3) "the item could not be assessed". The evaluators captured additional comments for checklist items and the overall app. We examined the data in two ways: 1) evaluators' overall scores: across all HLO checklist items and between evaluators, and 2) categorical scores: across all evaluators and between each HLO checklist categories.

\section{Results}

\subsection{HLO Checklist Results}

Each evaluator's overall percentage of "yes" scores on checklist items ranged from $60.0 \%-65.7 \%$ and "no" scores ranged from $28.6 \%-37.1 \%$. None of the evaluators could confidently assess the accessibility features. Although it was possible to change font size, this was achieved by configuring settings at operating system (OS) level; not within the app. Additionally, there was no text to speech feature. As seen in Figure 1, the app scored highest in the Organize Content and Simplify Navigation category (average 83\%) and lowest in the Engage Users category (average 33\%). For specific examples of strengths and weaknesses in each category, see Table 1.

\subsection{User Experience and Usability}

The evaluators identified several germane usability issues that were not captured by the HLO checklist. For example, one of the primary strengths of this app was that it did not require users to set up an account, only provide basic profile information, and emphasized users' data protection. However, not having an account meant the app was not password protected, which could be problematic on shared devices. In addition, the scanning feature was inoperative, yet failed to provide cues to indicate it was unavailable. This could have been a pivotal feature to improve usability and the efficiency of data entry. Instead, users entered data manually - a tedious and error prone exercise. 


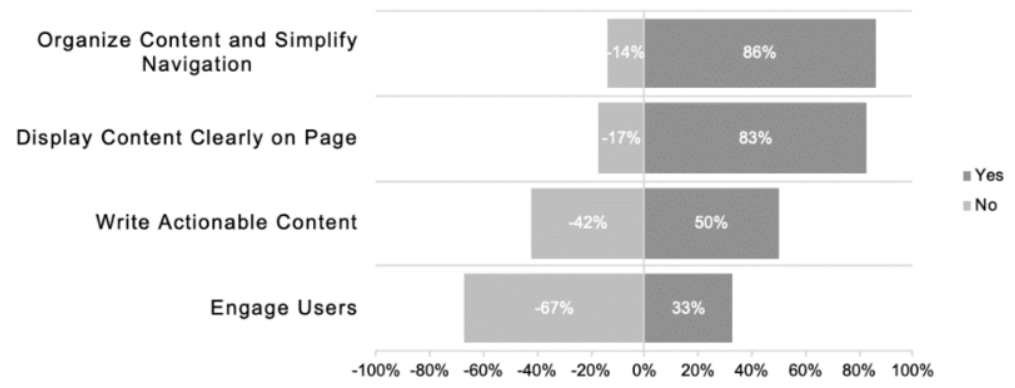

Figure 1. Percentage of HLO Checklist Items Satisfied (+= yes) and Unsatisfied (- = No) by Category. Note: the "missing" 8\% from Write Actionable Content is due to accessibility not being assessed.

Table 1. Examples of Strengths and Weaknesses in Each HLO Checklist Category

\begin{tabular}{lll}
\hline \multicolumn{1}{c}{ Category } & \multicolumn{1}{c}{ Strength } & \multicolumn{1}{c}{ Weakness } \\
\hline $\begin{array}{l}\text { Organize Content and } \\
\text { Simplify Navigation }\end{array}$ & $\begin{array}{l}\text { Search feature for manual entry. } \\
\text { Results clearly displayed and } \\
\text { values were color coded. }\end{array}$ & $\begin{array}{l}\text { No "Back" button. Instead, the home } \\
\text { button functions as a back button instead } \\
\text { of a home button. }\end{array}$ \\
\hline $\begin{array}{l}\text { Display Content Clearly } \\
\text { on the Page }\end{array}$ & $\begin{array}{l}\text { Links are obvious and intuitive. } \\
\text { Headings are meaningful and } \\
\text { have complementary icons. } \\
\text { Ample whitespace and contrast. }\end{array}$ & $\begin{array}{l}\text { Content cannot be shared or printed. } \\
\text { App does not adjust to landscape } \\
\text { orientation. }\end{array}$ \\
\hline $\begin{array}{l}\text { Write Actionable } \\
\text { Content }\end{array}$ & $\begin{array}{l}\text { Provided specific action steps. } \\
\text { Provides additional references. }\end{array}$ & $\begin{array}{l}\text { Important information is located below } \\
\text { the fold after less pertinent information. } \\
\text { Instances of using laboratory and } \\
\text { medical jargon. }\end{array}$ \\
\hline Engage Users & Easy to enter results into forms. & $\begin{array}{l}\text { Lacks intuitive, interactive tools, and } \\
\text { visualizations. } \\
\text { Does not offer multimedia information. }\end{array}$ \\
& &
\end{tabular}

The evalators identified several critical usability issues related to manual entry. First, we believe the additional workload and time required for data entry would create barriers to adoption. Second, the software did not provide enough instruction and support to assist users struggling with the on-screen affordances (e.g., no suggested alternatives for spelling errors, no results for "Urine Ph") for entering in technical terms. Third, we suspect users might forego inputting all data from a report, potentially affecting the comprehensiveness and value of the output. Finally, manual entry is inherently data prone and we forecast that transcription errors will be a common occurrence. For example, for RBC users were presented with many unit options and has to interpret that the unit " $\mathrm{E}$ E12/L" is equivalent to $\mathrm{x} 10^{12} / \mathrm{L}$. Another manual entry usability problem was related to the lack of system feedback after the user entered each result: there was there was no indication an entry was added to the report, causing confusion or the creation of multiple separate reports for each test rather than a single report with multiple tests.

Another problem identified was that the gender and birthdate were prepopulated, which could result in entering or using inaccurate data, and therefore alter the interpretation of the lab test result. With regards to gender, the application uses the term "Gender," in reference to sex, and only provides options for "Female" or "Male," therefore, reducing its inclusivity. Finally, the app crashed every time a user attempted to change the weight units from kilograms to pounds. 


\section{Discussion and Conclusions}

We evaluated a novel mobile app for citizens that automatically converts lab results from a standard medical report into a more suitable format (e.g., visualizing results, using plain language descriptions of tests, providing advice for next steps). Although the app had its shortcomings, we see it as an important step towards providing citizen-centred lab results and a vast improvement over traditional designs. The app's concept holds merit and when the software worked, the output was understandable and usable. Despite its promise, the app suffered in many usability dimensions (e.g., ease-of-use, error tolerance, accessibility, embedded help messaging and support). Ideally, data should be automatically extracted and converted. For these reasons, we do not endorse widescale deployment or even more robust simulation testing yet. This research provided several important insights that may be generalizable to other consumer health technologies. We also demonstrated that the HLO Checklist [11] framework can be applied using a systematic, team-based approach. Usability and eHealth literacy are inextricable in consumer health informatics [14]. For the field of health informatics to support citizen empowerment and participation in their own health care, tools need not only have content that is easy to understand and apply, but tools must be enjoyable and usable.

\section{References}

[1] Leckart S. The Blood Test Gets a Makeover. WIRED. 2010, Available at https://www.wired.com/2010/11/ff_bloodwork/, Accessed Jan 13, 2021.

[2] Reference Ranges and What They Mean Lab Tests Online, Available at https://labtestsonline.org/articles/laboratory-test-reference-ranges, Accessed Mar 7, 2021.

[3] Canadian Council on Learning. Health literacy in Canada: A healthy Understanding. 2008 p. 38.

[4] Mahadevan R. Health Literacy Fact Sheets [Internet]. Center for Health Care Strategies. 2013, Available at https://www.chcs.org/resource/health-literacy-fact-sheets/, Accessed Jan 13, 2021.

[5] Sørensen K, Pelikan JM, Röthlin F, Ganahl K, Slonska Z, Doyle G, et al. Health literacy in Europe: comparative results of the European health literacy survey (HLS-EU). Eur J Public Health. 2015 Dec;25(6):1053-8.

[6] Kelman A, Robinson C, Cochin E, Ahluwalia N, Braverman J, Chiauzzi E, et al. Communicating laboratory test results for rheumatoid factor: what do patients and physicians want? Patient Prefer Adherence. 2016 Dec;10:2501-17.

[7] Nystrom DT, Singh H, Baldwin J, Sittig DF, Giardina TD. Methods for Patient-Centered Interface Design of Test Result Display in Online Portals. EGEMs Gener Evid Methods Improve Patient Outcomes. 2018 Jun 26;6(1):15.

[8] Zikmund-Fisher BJ, Scherer AM, Witteman HO, Solomon JB, Exe NL, Tarini BA, et al. Graphics help patients distinguish between urgent and non-urgent deviations in laboratory test results. J Am Med Inform Assoc JAMIA. 2017 May 1;24(3):520-8.

[9] Cimino JJ, Patel VL, Kushniruk AW. The patient clinical information system (PatCIS): technical solutions for and experience with giving patients access to their electronic medical records. Int J Med Inf. 2002 Dec;68(1-3):113-27.

[10] Zikmund-Fisher BJ, Fagerlin A, Ubel PA. Improving understanding of adjuvant therapy options by using simpler risk graphics. Cancer. 2008 Dec 15;113(12):3382-90.

[11] Checklist - Health Literacy Online | health.gov, Available at https://health.gov/healthliteracyonline/checklist/, Accessed Jan 2, 2021.

[12] Monkman H, Schmidt T, Nøhr C. Online Medication Information for Citizens: A Comparison of Demands on eHealth Literacy. Stud Health Technol Inform. 2020 Jun 16;270:1026-30.

[13] Walsh L, Hemsley B, Allan M, Adams N, Balandin S, Georgiou A, et al. The E-health Literacy Demands of Australia's My Health Record: A Heuristic Evaluation of Usability. Perspect Health Inf Manag. 2017;14(Fall):1f.

[14] Monkman H, Kushniruk AW. The Consumer Health Information System Adoption Model. Stud Health Technol Inform. 2015;218:26-31. 